\title{
The innate immune function of airway epithelial cells in inflammatory lung disease
}

\author{
Pieter S. Hiemstra', Paul B. McCray $\mathrm{Jr}^{2}$ and Robert Bals ${ }^{3}$
}

\begin{abstract}
Affiliations:
${ }^{1}$ Dept of Pulmonology, Leiden University Medical Center, Leiden, The Netherlands.

${ }^{2}$ Dept of Pediatrics, Carver College of Medicine, University of lowa, lowa City, IA, USA.

${ }^{3}$ Dept of Internal Medicine V - Pulmonology, Allergology and Critical Care Medicine, Saarland University, Homburg, Germany.
\end{abstract}

\section{Correspondence:}

Pieter S. Hiemstra, Dept of Pulmonology, Leiden University Medical Center, P.0. Box 9600, 2300 RC Leiden, The Netherlands.

E-mail: p.s.hiemstradlumc.nl

ABSTRACT The airway epithelium is now considered to be central to the orchestration of pulmonary inflammatory and immune responses, and is also key to tissue remodelling. It acts as the first barrier in the defence against a wide range of inhaled challenges, and is critically involved in the regulation of both innate and adaptive immune responses to these challenges. Recent progress in our understanding of the developmental regulation of this tissue, the differentiation pathways, recognition of pathogens and antimicrobial responses is now exploited to help understand how epithelial cell function and dysfunction contributes to the pathogenesis of a variety of inflammatory lung diseases. Herein, advances in our knowledge of the biology of airway epithelium, as well as its role and (dys)function in asthma, chronic obstructive pulmonary fibrosis and cystic fibrosis will be discussed.

@ERSpublications

Discussing innate immune mechanisms active in airway epithelial cells relevant for asthma, COPD and cystic fibrosis http://ow.ly/HCsiG

Received: Aug 012014 | Accepted after revision: Dec 152014 | First published online: Feb 192015

Support statement: P.S. Hiemstra was supported by grants from the Lung Foundation Netherlands (Amersfoot, the Netherlands), Grifols (Frankfurt am Main, Germany) and Galapagos NV (Mechelen, Belgium). P.B. McCray Jr was supported by grants NIH HL-51670, HL-091842 and HL-118000, and received support from the Roy J. Carver Charitable Trust (Muscatine IA, USA). R. Bals received support from the Deutsche Forschungsgemeinschaft (Bonn, Germany) (Ba 1641/12) and Bundesministerium für Forschung (Bonn) (01Gl1001, 01 GM111 OB, 01 Kl1 01 OC).

Conflict of interest: Disclosures can be found alongside the online version of this article at erj.ersjournals.com

Copyright OERS 2015 


\section{Introduction}

Airway epithelial cells (AECs) are located at the interface between the external and internal milieu, and are exposed to an array of inhaled gases and particles on a daily basis. The classic view of the airway epithelium is that of a structural barrier that regulates water and ion transport, and contributes to the clearance of inhaled substances through mucociliary clearance. However, recent research reveals that the airway epithelium is highly dynamic and displays a broad spectrum of activities related to inflammation, immunity, host defence and tissue remodelling. This article focuses on the role of the airway epithelium in innate immunity and host defence against bacterial and viral infections in health, as well as in asthma, chronic obstructive pulmonary disease (COPD) and cystic fibrosis (CF).

\section{Development and structure of the airway epithelium}

AECs line the airways and serve as a means of transportation of gases to and from the alveoli. They are the most abundant cell type in the lung and the first to encounter inhaled substances; therefore, they are important in the regulation of host defence. Inhaled air contains numerous substances, including toxic compounds, microorganisms and microbial products that may cause pulmonary injury. The airway epithelium contributes to defence by a variety of mechanisms that are discussed in more detail later. Briefly, these mechanisms include the barrier function of the epithelium and mucociliary clearance, as well as the production of antimicrobial peptides (AMPs) and proteins, reactive oxygen and nitrogen species (ROS and RNS, respectively), and a range of cytokines, chemokines and growth factors $[1,2]$. This allows the airway epithelium to directly contribute to host defence, and augment the response via the recruitment of a range of leukocytes and communication with mesenchymal cells in the airway wall. This intense communication network involves not only soluble mediators but also cell-cell contacts, and allows AECs to transfer a signal resulting from exposure to inhaled substances to other cells, and thus instruct adaptive immunity.

In recent years, significant progress has been made in our understanding of lung development, including the airway epithelium. This is important for understanding the innate immune function of the airway epithelium, because it is increasingly recognised that early life events during development contribute to adult lung disease. Furthermore, lung development research has resulted in new tools to study epithelial cell function in inflammatory lung disease. Indeed, recently, the ability to generate AECs from human embryonic stem cells and induced pluripotent stem cells has contributed to the development of the airway epithelium, and will probably continue to do so [3-5]. Excellent recent reviews on lung development and lung stem/progenitor cells are available [6-8].

The pseudostratified airway epithelium of newborns and adults is composed of a variety cell types, including basal cells, ciliated cells, mucus-producing goblet cells, club cells and neuroendocrine cells. Submucosal glands are mainly located in the large airways and are composed of terminal serous and mucous cells and the collecting duct cells. The balance of these various cell types differs between the trachea, bronchi and the more distal airways where, for example, goblet cells are normally nearly absent. However, the composition of the epithelium is clearly altered in asthma, COPD and CF, with an increase in goblet cells and obstruction by mucus, especially in the small airways. The epithelial layer forms a tight barrier, separating the lumen of the airways and its inhaled content from the underlying tissue. An essential component of this barrier is the luminal junctional complex, which is composed of apical tight junctions and adherens junctions. This complex is disturbed by inhaled substances such as pollutants, microorganisms and inhaled allergens, and is dysfunctional in a variety of airway diseases, including asthma and COPD. This impaired epithelial barrier function also impacts on susceptibility to respiratory infections.

Basal cells are progenitors that are capable of self-renewal and generation of all surface epithelium cell types, and are characterised in humans by expression of p63 and KRT14 [9]. Differentiated AECs can also dedifferentiate into stem cells and, thus, contribute to regeneration of the differentiated airway epithelium [10]. Epithelial cell development and differentiation is tightly regulated by epigenetic mechanisms, including histone modifications and microRNAs [7]. The discovery of microRNAs as regulators of post-transcriptional gene expression was one of the most exciting advances in biology during the late 20th century. MicroRNAs mediate changes in gene expression post-transcriptionally through translational repression or mRNA degradation [11]. Many lines of evidence now show that changes in microRNA actions can influence epithelial cell function in asthma, COPD and CF. Injury and repair, cell adhesion, cell proliferation, cell migration and differentiation are finely regulated by microRNAs. Several studies have identified roles for microRNAs in lung development [7, 12, 13] and disease states, including COPD [11] and asthma [14]. Their role in CF is discussed more extensively below. Therefore, microRNAs and other epigenetic mechanisms have a major effect on epithelial cell development and function. 


\section{AEC culture models}

Advances in basic and translational studies require suitable model systems to bridge the gap between heterologous cell populations and human studies. Investigations of AEC biology have benefited greatly from the development and continued refinement of primary cultures from surface epithelia of the trachea, bronchi and alveoli. An air-liquid interface (ALI) culture method of cells grown on a permeable filter with air above and cell culture media below has been widely employed [15]. The validity of this model is supported by studies of the ultrastructure, but also the function of the epithelium, including mucus secretion, airway surface liquid (ASL) regulation and mucociliary transport, as well as inflammatory and antimicrobial mediator production. This is supported by gene expression profiling experiments that have revealed remarkable similarities between the mRNA expression profiles of ALI-cultured epithelia and bronchoscopically obtained tracheal and bronchial brushings from human airways [16, 17]. These findings support the utility of this culture model and strengthen the rationale for its use as an experimental platform to complement in vivo studies. In addition, the ALI model is also used to generate primary epithelial cell cultures from genetically modified animals and, thus, helps to define the function of individual genes in epithelial cell function [18]. Developments in imaging and culture techniques are also of interest, including super-resolution microscopy techniques, epithelial cell and lung organoids, microfluidic lung-on-a-chip system and use of lung tissue slices in vitro. A significant recent advance is the application of a Rho kinase inhibitor and/or fibroblast feeder cells or conditioned media as a strategy to greatly expand primary cell populations by inhibiting cellular senescence, making cultures derived from small patient samples, such as nasal or bronchial brushings, even more feasible $[19,20]$.

Recent progress in the production of induced pluripotent stem cells from human and animal sources provides an opportunity for the creation of biobanks and expansion of disease and patient-specific cells. Several groups are making progress towards the goal of differentiating induced pluripotent stem cells into AECs [4, 5]. Further advances will greatly facilitate studies of cell therapies, tissue engineering, pharmacogenomics and personalised medicine. New gene targeting and gene repair technologies, including zinc finger nucleases, transcription activator-like effector nucleases and CRISPR-cas9 [21], provide further opportunities to generate isogenic models for investigations.

Although differentiated cultures derived from primary AECs and induced pluripotent stem cells do show remarkable similarities with AECs in tissue with regard to morphology, cell-specific markers and gene expression profiles, it needs to be noted that the cells in these model systems may differ from native tissue cells. In addition, it is important that results obtained using these models are confirmed using cells from multiple donors. This way full advantage can be taken from the superiority of these models compared to those based on immortalised or tumour cell lines.

\section{The microbiome of the lung}

A number of recent studies have shown that microorganisms are present in the lungs. AECs are continuously exposed to this distinct collection of bacteria, viruses, fungi and their products. The term "microbiome" is defined as the "ecological community of commensal, symbiotic and pathogenic microorganisms that literally share a specific site of the body" [22]. Currently, real-time PCR, pyrosequencing, restriction fragment length polymorphism analysis and direct sequencing are applied to analyse the microbiome. These approaches showed that the lung microbiome is complex in healthy individuals and altered in diseases. In asthma and COPD, the microbiome of the lung is different from healthy individuals and is probably mechanistically linked to disease processes $[23,24]$. This is confirmed by studies in mouse models of allergic airways inflammation showing a link between the development of the lung microbiome and immunological tolerance to allergens [25]. In CF lung disease, analysis of the lung microbiome revealed a complex composition, far beyond the current knowledge [26, 27]. Furthermore, the lung microbiome is dynamic and changes significantly after rhinovirus infection in COPD [28].

Analogous to the gut, the interaction between epithelial cells and the microbiome is probably an important factor in maintaining stable homeostasis [29]. In the lung, AECs are also in continuous contact with the microbiome and this interaction is critical to maintaining a sufficient barrier. Hypothetically, inhaled or aspirated microorganisms are quickly inactivated and killed, and their products interact with immune cells and AEC, and thus help shape immune responses [30].

\section{Sensing microbial presence}

The continuous threat posed by microbial exposure requires sensitive detection mechanisms, as well as scaling of responses to avoid unwanted inflammation and tissue injury. In 1989, using the pattern recognition theory, JANEWAY [31] proposed that the host innate immune system senses microbial presence through the detection of so-called pathogen-associated molecular patterns, which are invariant molecular structures that are present in pathogens but absent in the host. The identification of the Toll-like receptor (TLR) family 
of transmembrane proteins was the first step in delineating the pattern recognition receptors that bind and respond to pathogen-associated molecular pattern exposures. Subsequent studies also showed the presence of cytosolic receptors for sensing microbial presence. These and other mechanisms that contribute to microbial detection by AECs are discussed below (fig. 1).

\section{Pattern recognition receptors: TLRs, lectin-type receptors and intracellular receptors}

Pattern recognition receptors are germline encoded receptors with four current families: the TLRs, the C-type lectin receptors (CLRs), the cytoplasmic proteins retinoic acid-inducible gene-I-like (RLRs) and the NOD-like receptors (NLRs) [32]. Orthologous receptors have been identified in multiple species including drosophila, mouse and human [33]. The functions of TLRs in Drosophila comprise developmental regulation and host defence [34], while in mammals TLRs have a restricted role as pattern recognition receptors in innate immunity by recognising microbial and endogenous ligands. The 10 TLRs identified in humans and the 12 TLRs in mice are structurally characterised by N-terminal leucine-rich repeats, a transmembrane region and a cytoplasmic Toll/IL-1 receptor homology (TIR) domain that mediates signalling. The cellular localisation and ligands are summarised in table 1. TLR-mediated signalling is complex and involves MyD88 and TIR-domain-containing adapter-inducing interferon- $\beta$ (TRIF) [35]. Whereas AECs express most TLRs [36], they often seem to be hyporesponsive to microbial stimulation and alveolar macrophages are necessary to induce a full epithelial host defence reaction [37].

CLRs comprise a large number of proteins highly conserved in vertebrates that were originally characterised by a C-type lectin domain (mannose binding), but now encompass more structurally divergent molecules [38]. Dectin-1 is a CLR expressed in AECs and involved in the detection of mycobacteria and Aspergillus [39, 40]. RLRs are complex cytoplasmic receptors that recognise the presence of genomic RNA of dsRNA viruses or dsRNA intermediates from ssRNA viruses. Melanoma differentiation-associated gene-5 is expressed in AECs and involved in the detection of rhinovirus and other viruses [41]. Finally, NLRs are intracellular pattern recognition receptors that are characterised by a central nucleotide-binding and oligomerisation domain (NOD or NACHT) and C-terminal leucine-rich repeats [42]; limited information is available about NOD function in AECs.
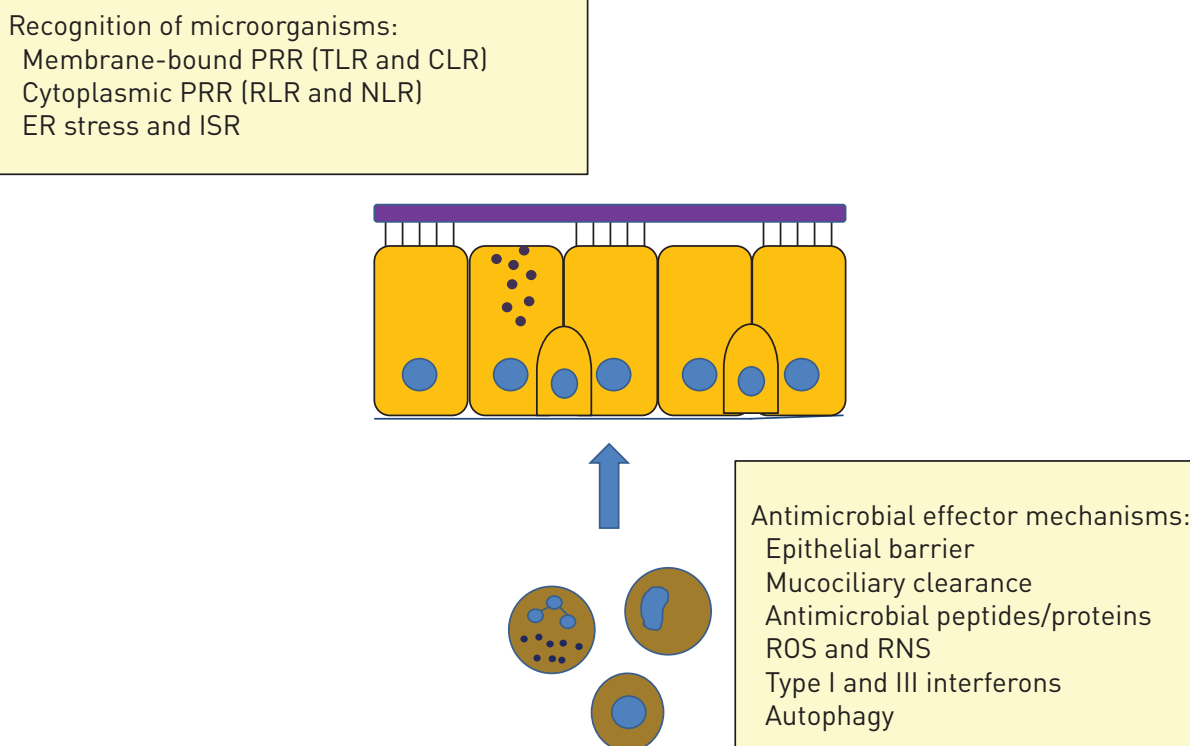

FIGURE 1 The innate immune function of the airway epithelium: sensing microbial presence and antimicrobial effector mechanisms. Various cell types are involved in the innate immune function of the airway epithelium, including basal cells, goblet cells, ciliated cells and club cells. Microbial presence is detected by pattern recognition receptors (PRR), i.e. membrane-bound Toll-like receptors (TLR), C-type lectin receptors (CLR), cytoplasmic retinoic acid-inducible gene-I-like receptors (RLR) and NOD-like receptors (NLR). Other mechanisms are also involved in this recognition process, including endoplasmic reticulum (ER) stress and the integrated stress response (ISR). Antimicrobial effector mechanisms include the barrier function of the epithelium, mucociliairy clearance and the antimicrobial activity of mucus, antimicrobial peptides, reactive oxygen species (ROS) and reactive nitrogen species (RNS), antiviral interferons (type I and III interferons) and autophagy. In addition, production of cytokines, chemokines and other mediators results in the recruitment of cells of the adaptive and innate immune system, which may contribute to host defence. 
TABLE 1 Toll like receptors (TLRs) of humans and mice

\begin{tabular}{lcc} 
TLR & Cellular localisation & Microbial ligand \\
\hline TLR1 & Plasma membrane & Lipoproteins \\
TLR2 & Plasma membrane & Lipoproteins \\
TLR3 & Endosome & Double-stranded RNA \\
TLR4 & Plasma membrane & Lipopolysaccharide \\
TLR5 & Plasma membrane & Flagellin \\
TLR6 & Plasma membrane & Diacyl lipoprotein \\
TLR7 & Endosome & Single-stranded RNA \\
TLR8 & Endosome & Small synthetic compounds; \\
& & single-stranded RNA \\
TLR9 & Endosome & CpG-oligonucleotides \\
TLR10 & Endosome & Putatively influenza-related ligand \\
TLR11, 12, 13 (mouse not humans) & Endosome & Profilin-like molecule (parasites)
\end{tabular}

Integrated stress response and endoplasmic reticulum stress

The integrated stress response (ISR) can be activated by one of four stress-sensing kinases, which are triggered by a variety of signals including those generated during infection [43]. These kinases cause phosphorylation of the $\alpha$-subunit of the eukaryotic translation initiation factor 2 (eIF2 $\alpha$ ), resulting in the inhibition of protein synthesis which may be cytoprotective, e.g. by inhibiting viral replication. eIF $2 \alpha$ phosphorylation also causes translation of selected mRNAs, including the transcription factor ATF4, which results in enhanced expression of genes involved in adaptation to cellular stress. The kinase PKR is an example of a stress-sensing kinase that causes phosphorylation of eIF $2 \alpha$ during infection, since it is triggered by dsRNA present during viral infection. The kinase PERK senses protein (mis)folding in the endoplasmic reticulum and is activated during endoplasmic reticulum stress, which may result from the increased demand on the endoplasmic reticulum during infection as a result of production of, for example, AMPs and pro-inflammatory mediators. Thereby, PERK and the downstream signalling, that is shared with three other kinases of the ISR, comprises one of the three arms of the unfolded protein response to endoplasmic reticulum stress [44]. The crosstalk between immune activation, for example by TLRs and the responses to cellular stress by the unfolded protein response, and ISR provides evidence for the numerous possibilities for fine-tuning the epithelial response to infection [45]. Whereas acute activation of the ISR and the unfolded protein response may contribute to cell survival and host defence against infection, chronic activation as observed in COPD and CF may be detrimental [43].

Integration of signals: scaling of danger

The availability of this variety of receptors and mechanisms to detect microbial presence and the ability to integrate this information allows the innate immune system to scale responses [46]. Recent studies demonstrating that production of AMPs by upper airway AECs is triggered by bitter receptors (T2R), a response that is blocked by sweet taste receptors (T1R2/3), provides additional information on the regulation of innate immunity at the epithelial surface [47]. This is based on the hypothesis that glucose consumption by microorganisms at sites of infection may reverse this inhibition by T1R $2 / 3$ receptors, which is in line with the hypothesis that detection of microbial viability is an important means to scale the response.

\section{Epithelial effector mechanisms in host defence against infection}

A variety of antimicrobial mechanisms contribute to clearance of microorganisms that are a potential threat to the host (fig. 1). The vast redundancy in the system prevents microbial resistance, although many mechanisms are most effective against bacteria in their planktonic phase, and less active against bacteria in biofilms.

\section{Mucins}

Mucus is an important component of mucociliary clearance and is an extracellular gel comprised of water, mucins and numerous associated molecules [48]. Mucins are large glycoproteins released by several secretory cells, including goblet cells, club cells and the serous and mucous cells of the glands [49]. Mucin proteins are polydispersed in size (2-50 MDa) and display a random coil formation in solution, stabilised by disulphide-linked subunits. Large carbohydrate chains are attached to the protein backbone. Mucins are encoded by 17 MUC genes: 10 code for cell-tethered mucins (MUC1, MUC3A, MUC3B, MUC4, MUC12, MUC13, MUC16, MC16, MUC17 and MUC20), five code for secreted mucins (MUC2, MUC5A/C, MUC5B, MUC6 and MUC19) and two code for nonpolymeric glycoproteins (MUC7 and MUC8) [49]. 
The mucin content of airway mucus is mainly characterised by glycoproteins encoded by MUC5AC, MUC5B and MUC2. Amongst these airway mucins, an important role of Muc5b in mucociliary clearance and host defence in the murine airways was recently described [50]. Mucin gene expression is regulated by a variety of factors including inhaled toxins or microorganisms, inflammatory cytokines and ErbB-receptor ligands. After synthesis, mucins are stored in condensed granules and their release is tightly controlled by calcium, ATP (via apical $\mathrm{P}_{2} \mathrm{Y}_{2}$ receptors) and other mechanisms [51].

A number of smaller molecules are associated with mucus, mediated by charge interactions between these molecules and the negatively charged mucins. Many of these proteins display antimicrobial activity, including secretory IgA, AMPs, lysozyme and collectins.

The current view is that a discontinuous mucus layer is located above the periciliary space with the tips of the cilia reaching the mucus. It is separated from the epithelial surface by the periciliary layer or sol layer, in which macromolecules (cell-tethered mucins and glycosaminoglycans) are attached to the cilia and form a protected area that cannot be penetrated from above by mucus or other molecules [52]. Together the mucus layer and periciliary layer comprise the ASL, and its structure and properties are controlled by different mechanisms, including transepithelial ion and water transport. Ion channels, such as the cystic fibrosis transmembrane conductance regulator (CFTR) and epithelial sodium channel (ENaC), regulate the volume and composition of airway secretions [53].

\section{Mucociliary transport}

The coordinated beating of cilia interacting with mucus provides an important mechanism for clearance of inhaled or aspirated particulates or microbes via mucociliary transport. Decreased clearance of pathogens and inflammatory mediators results in inflammation, infection and tissue destruction. In CF, loss of CFTR function reduces ASL $\mathrm{pH}$, impairs liquid secretion and causes mucus strands to remain tethered to submucosal gland ducts resulting in reduced mucociliary transport and altered host defence [54, 55].

\section{Antimicrobial peptides}

AMPs are small peptides $(\sim 10-50$ amino acids) that have antimicrobial activity against bacteria, viruses and fungi. In addition, many of them probably act as modulators of inflammation, repair, regeneration and other processes. In a narrow view, AMPs are gene encoded; however, in a broader view, AMPs also arise from proteolytic fragments of larger proteins. AECs of mammals produce AMPs of the defensin and cathelicidin families.

The structural hallmark of defensins is the presence of six cysteines that form three intramolecular disulfide bonds. Based on their structure, defensins can be subdivided into $\alpha$-, $\beta$ - or $\theta$-defensins. While $\alpha$-defensins are expressed in myeloid cells and $\theta$-defensins are not functionally expressed in humans, AECs produce $\beta$-defensins. Human $\beta$-defensin 1, 2, 3 and 4 are mainly expressed in AECs, synthesised as precursors and secreted after cleavage from the propiece. While human $\beta$-defensin-1 (hBD-1) appear to be secreted constitutively, the production of other $\beta$-defensins is induced by various signalling pathways, such as the TLR or nuclear factor- $\kappa \mathrm{B}$ pathways, or the presence of proinflammatory cytokines. Interestingly, the number of defensin genes varies between individuals from two to 12 (copy number variants). The number of defensin gene clusters that are present modulates disease outcomes for psoriasis, but no correlation has been confirmed for COPD or asthma [56]. The antimicrobial activity of defensins is probably based on interactions with microbial lipids and covers Gram-positive and Gram-negative bacteria, viruses and fungi. In addition to their antimicrobial function, $\beta$-defensins were shown to chemoattract immune cells and activate dendritic cells. The role of $\beta$-defensins in the lung is not entirely clear. Murine knockout models did not provide a clear answer due to the redundancy of these AMPs and indicated a host defence function $[57,58]$. In human disease, acute inflammation seems to increase defensin expression, while chronic inflammation may suppress expression of these AMPs [59].

The cathelicidins are a large group of AMPs characterised by the homologous propiece, called "cathelin", which acts as inhibitor of cathepsin L. The structures of the C-terminal active peptide differ amongst species. In humans and mice, only one cathelicidin gene is present and encodes the peptides hCAP-18/ LL-37 (gene name CAMP) or CRAMP (gene name Camp). Both peptides are linear molecules that have an $\alpha$-helical structure in physiological solution. These peptides undergo post-translational modification and have broad activity against various microorganisms, including viruses. In the lung, AECs, neutrophils, macrophages and other cells produce LL-37 [60]. The biological role in pulmonary host defence has been shown using the CRAMP knockout model [61]. In addition, cathelicidins appear to have a complex and broad role in the modulation of diverse biological processes mediated through specific receptors or through nonspecific interactions with biomembranes [62]. Cathelicidins have a role in angiogenesis, wound healing of epithelia, lung cancer growth and regulation of immune cells. A major source of LL-37 in the lung is the neutrophil, while vitamin D induces the peptide's expression in macrophages and AECs [63]. 
In addition to defensins and cathelicidins, numerous other proteins or peptides with antibacterial activity are secreted from AECs, including lysozyme, lactoferrin and secretory leukocyte proteinase inhibitor. Most of these host defence molecules are positively changed and, thus, interact closely with negatively charged macromolecules. As outlined above, mucus [64] and neutrophil extracellular traps (suggested as host defence factors) [65] contain high concentrations of cationic host defence peptides. Interactions between AMPs and components released during inflammation and tissue injury (e.g. F-actin and DNA) probably inhibit host defence activities.

\section{Miscellaneous mechanisms}

ROS and RNS

AEC produce substantial amounts of ROS, mainly arising from the NADPH oxidases DUOX1 and DUOX2. DUOX-derived ROS has been shown to contribute to the antimicrobial activity that is generated by AECs in the ASL. This results from the formation of antimicrobial OSCN ${ }^{-}$from DUOX-derived $\mathrm{H}_{2} \mathrm{O}_{2}$ catalysed by lactoperoxidase [66], a system that may be defective in the airways of CF patients because of a deficient secretion of $\mathrm{SCN}^{-}$by the CF airway epithelium [67]. In addition to ROS, AECs also produce RNS through the action of nitric oxide synthases (NOS) enzymes, and the resultant nitric oxide displays a variety of functions in immune regulation and host defence against infection [68]. Nitric oxide production in the airways is derived from the constitutive NOS- 1 and NOS-3 enzymes, and the inducible NOS- 2.

\section{Antiviral interferons}

A variety of the aforementioned mechanisms contribute to antiviral defences of the airway epithelium, including mucins, antimicrobial peptides and RNS/nitric oxide. Detection of viral infection by the aforementioned membrane-bound and intracellular recognition mechanisms also triggers the production of type I interferons (IFN- $\alpha$ and $-\beta$ ) and type III interferons (IFN- $\lambda$ ) [69]. Interferons induce the expression of a range of genes encoding proteins that interfere with viral replication, protein synthesis and trafficking. In diseases including COPD, asthma and CF, interferon-mediated host defences may be blunted [70]. Based on these findings, novel drugs are being developed that are not directly antiviral, but cause activation of IFN signalling pathways and thus enhance antiviral defences [71]. Furthermore, in CF, impaired interferon-mediated STAT1 signalling and impaired induction of antiviral inducible NOS2 and OAS1 may also contribute to increased epithelial susceptibility to infection by respiratory viruses [72].

\section{Autophagy}

Autophagy is a homeostatic mechanism that delivers unwanted cellular components to lysosomes for degradation. It plays a role in cell stress, differentiation and development, as well as the clearance of toxic components and (intracellular) pathogens [73]. In contrast, excessive and uncontrolled autophagy is detrimental to the host and contributes to the pathogenesis of COPD, for example [74].

\section{Epithelial cell (dys)function in inflammatory lung diseases \\ Asthma}

Asthma is characterised by airways inflammation, structural alterations in lung tissue, variable airflow limitation and airway hyperresponsiveness. In the most common form of asthma, atopic allergen-induced asthma, eosinophils and T-helper cell (Th)2 lymphocytes play central roles. Genetic studies have provided insights into the role of gene-environment interactions, and it is interesting to note that a substantial number of the genes associated with asthma, including PCHD1, IL-33 and ORMDL3, are expressed in the airway epithelium [75]. Asthma is accompanied by extensive alterations in the airway epithelium, including increased fragility, decreased barrier function, impaired anti-oxidant activity, increased goblet cells and impaired antiviral innate responses [76]. Furthermore, the important function of the airway epithelium in regulating fluid and ion transport is altered in asthma. It was found that Th2 cytokines increase epithelial expression of chloride channels, such as TMEM16A and SLC26A9, and that chloride secretion through these channels helps to prevent mucus obstruction of the airways $[77,78]$. In line with this, a single nucleotide polymorphism in SLC26A9 that is associated with reduced protein expression of the chloride channel is associated with asthma [78].

Infections play an important role in asthma, and rhinovirus infections are especially associated with asthma exacerbations. Studies on the human airway microbiome have also confirmed the involvement of bacterial colonisation and/or infection in the pathogenesis of asthma [79]. Increased susceptibility to infection can be explained by the: 1) decreased barrier activity of the airway epithelium in asthma; 2) decreased mucociliary clearance resulting from excessive mucus production, but also decreased epithelial innate immune responses to respiratory viruses [80]; and 3) inhibitory effect of Th2 cytokines on the production of antimicrobial peptides [81]. These respiratory infections contribute to inflammation, and 
have been suggested to also induce a "memory pool" of basal progenitor cells in the epithelium that, for example, produce excessive amounts of IL-33 [82].

It has long been thought that inflammation is a main driver of airway remodelling in asthma. However, inhalation of a bronchoconstrictor in patients with mild atopic asthma also resulted in an increase in measures of airway remodelling and an increase in epithelial transforming growth factor (TGF)- $\beta_{1}$ [83]. This suggests that mechanical forces exerted during bronchoconstriction cause stress in the airway epithelium resulting in airway remodelling. However, this increased epithelial expression of TGF- $\beta_{1}$ may also affect host defence. TGF- $\beta$ can inhibit production of the antimicrobial serine protease inhibitor secretory leukocyte proteinase inhibitor by AEC [84] and promotes rhinovirus replication in AEC [85]. However, TGF- $\beta$ may also have beneficial effects by counteracting the cigarette-smoke induced disruption of epithelial barrier function [86] and by suppressing epithelial mucin production induced by nontypeable Haemophilus influenzae (via suppression of p38 mitogen-activated protein kinase) [87] or IL-13 (an isoform-specific effect of TGF- $\beta_{2}$ ) [88].

\section{COPD}

Asthma and COPD share an increased susceptibility to respiratory infections. In COPD and smokers without COPD, a variety of studies have shown that cigarette smoke exposure impairs host defence by decreasing epithelial barrier function, ciliary function, antimicrobial peptide production and antiviral responses, while increasing mucus production $[59,76,89]$. Cigarette smoke is the main risk factor for development of COPD, and many of these epithelial features of COPD can be explained by a direct or indirect result of cigarette smoke exposure. Exposure of cultured AECs to cigarette smoke increases inflammatory mediator release and decreases barrier function [90] and expression of antimicrobial peptides [59]. Furthermore, smoke exposure also increases citrullination of the antimicrobial peptide LL-37, resulting in impaired antimicrobial and increased pro-inflammatory activity of this peptide [91]. These observations show that cigarette smoke increases inflammation, while decreasing host defence against infections, which is compatible with what is observed in COPD patients. Cigarette smoke may affect epithelial cell functions through a variety of mechanisms, including direct oxidant activity and TLR signalling, but may also induce endoplasmic reticulum stress and activate the ISR $[43,92]$. These latter findings are supported by studies in lung tissue from COPD patients [93]. Another feature of COPD that has recently been observed in lung tissue and culture is the impaired epithelial CFTR function and expression that was found to be associated with COPD and smoking [94]. Interestingly, as also observed in asthma and CF, epithelial features may persist after culturing AEC. This is shown by various studies, including that of SCHULZ et al. [95] who demonstrated that AECs from COPD patients release more IL- 8 than cells from smoking control subjects. AECs from patients with $\alpha_{1}$-antitrypsin deficiency, the main genetic risk factor for COPD, also display a proinflammatory phenotype in culture [96]. HoltzMan et al. [70] provided new insights into how infections may promote chronic inflammation in the airways. The authors showed that viral infection may activate basal cells, leading to a population of long-lived basal cells with increased expression of IL-33. IL-33 expression in this population was associated with increased IL-13 and mucin expression in mouse models of viral infection and in severe COPD [82].

\section{Cystic fibrosis}

The early pathogenic steps in CF lung disease have been difficult to elucidate because they occur in infants and pre-school-aged children. Mouse models of loss of CFTR function have not resulted in spontaneous lung disease with similarities to humans. To overcome this limitation, groups have developed new animal models by disrupting the CFTR gene in pigs [97, 98], ferrets [99] and rats [100]. Both CF pigs and ferrets spontaneously developed lung disease with several similarities to children with CF, including a mucosal host defence defect, ineffective eradication of bacteria, bacterial colonisation, increased mucus production and airway remodelling [101-103].

Experimental findings in these animal models are stimulating bench-to-bedside-and-back studies that have provided new insights into early steps in lung disease onset. For example, as a consequence of reduced bicarbonate transport due to loss of CFTR, the ASL of newborn CF pigs has a lower $\mathrm{pH}$ than littermate controls [54]. The reduction in ASL $\mathrm{pH}$ impairs the function of resident host defence peptides and proteins and reduces the antimicrobial activity of airway secretions. The ASL pH differences between non-CF and CF subjects may vary depending on age and disease state. McShane et al. [104] found no differences in ASL $\mathrm{pH}$ between people with $\mathrm{CF}$ and non-CF controls aged $\geqslant 3$ years. In contrast, ABOU ALAIWA et al. [105] recently reported that babies with CF had a lower nasal ASL pH compared to non-CF neonates, while nasal $\mathrm{pH}$ values in older $\mathrm{CF}$ children and adults were similar to values obtained in non-CF subjects. Together, these findings raise the possibility that interventions aimed at increasing ASL pH in the neonatal airways might improve host defence [106]. 
Impaired host defence against respiratory infections may result not only from the reduced activity of ASL antimicrobials, but also from a decreased mechanical clearance of inhaled pathogens. It is also widely believed that loss of CFTR function leads to amiloride-sensitive $\mathrm{Na}^{+}$hyperabsorption via $\mathrm{ENaC}$, depletion of the periciliary liquid layer, dehydration of the airways and impaired mucociliary clearance [107, 108]. However, recent findings in new CF animal models call into question whether sodium hyperabsorption is a primary early event in CF that contributes to decreased mucociliary transport at the time of disease onset. In line with findings in adult CF patients, newborn CF pigs exhibited impaired mucociliary transport under conditions of cholinergic stimulation [55]. However, this reduced mucociliary transport was not associated with sodium hyperabsorption or periciliary liquid depletion. Remarkably, the mucus released from CF submucosal glands was anchored to gland ducts. These findings were present at birth, in the absence of infection or inflammation, and indicate that impaired mucociliary transport is a primary defect in CF. Interestingly, airway epithelia in newborn CF pigs do not hyperabsorb sodium [109]. Additionally, experiments in neonatal CF ferrets [103, 110] and CF rats [100], and some results in human CF airway epithelia [111], similarly found no evidence of sodium hyperabsorption. These results suggest that loss of CFTR function is not associated with increased $\mathrm{ENaC}$ activity at the time of disease onset.

It remains possible that over time secondary disease-associated changes in the airways lead to sodium hyperabsorption. Sodium hyperabsorption can alter airway function as shown in transgenic mice overexpressing $\beta$-ENaC. Transgenic mice overexpressing $\beta$-ENaC develop a lung disease characterised by ASL volume depletion, mucus obstruction, chronic inflammation and structural lung damage [112]. Impaired mucociliary transport in patients with established CF lung disease might result from ASL volume depletion secondary to impaired or absent CFTR function and resulting in higher mucin hyperconcentration. This concept is supported by a recent study demonstrating higher mucin concentrations in secretions of adults with CF [113]. The investigators posit that the predicted increased osmotic pressures of CF mucus results in osmotic compression of the periciliary liquid layer in CF airways.

The post-translational regulation of CFTR expression and function by microRNAs is now established. Oglesby et al. [114] reported an increase in miR-494 levels in bronchial epithelial brushings from human CF airways. Repression of CFTR by miR-494 has also been reported [115, 116]. The reasons for dysregulated microRNA expression in CF are unknown. Cellular responses to inflammation, infection, cytokines, other inflammatory mediators or other changes in the ASL environment probably contribute. Additionally, RAMACHANDRAN et al. [117] demonstrated that human airway epithelia transfected with the mimics of miR-509-3p or -494 exhibited reduced CFTR expression and their respective anti-miRs had the opposite effect. Interestingly, both microRNAs acted cooperatively in regulating CFTR expression and

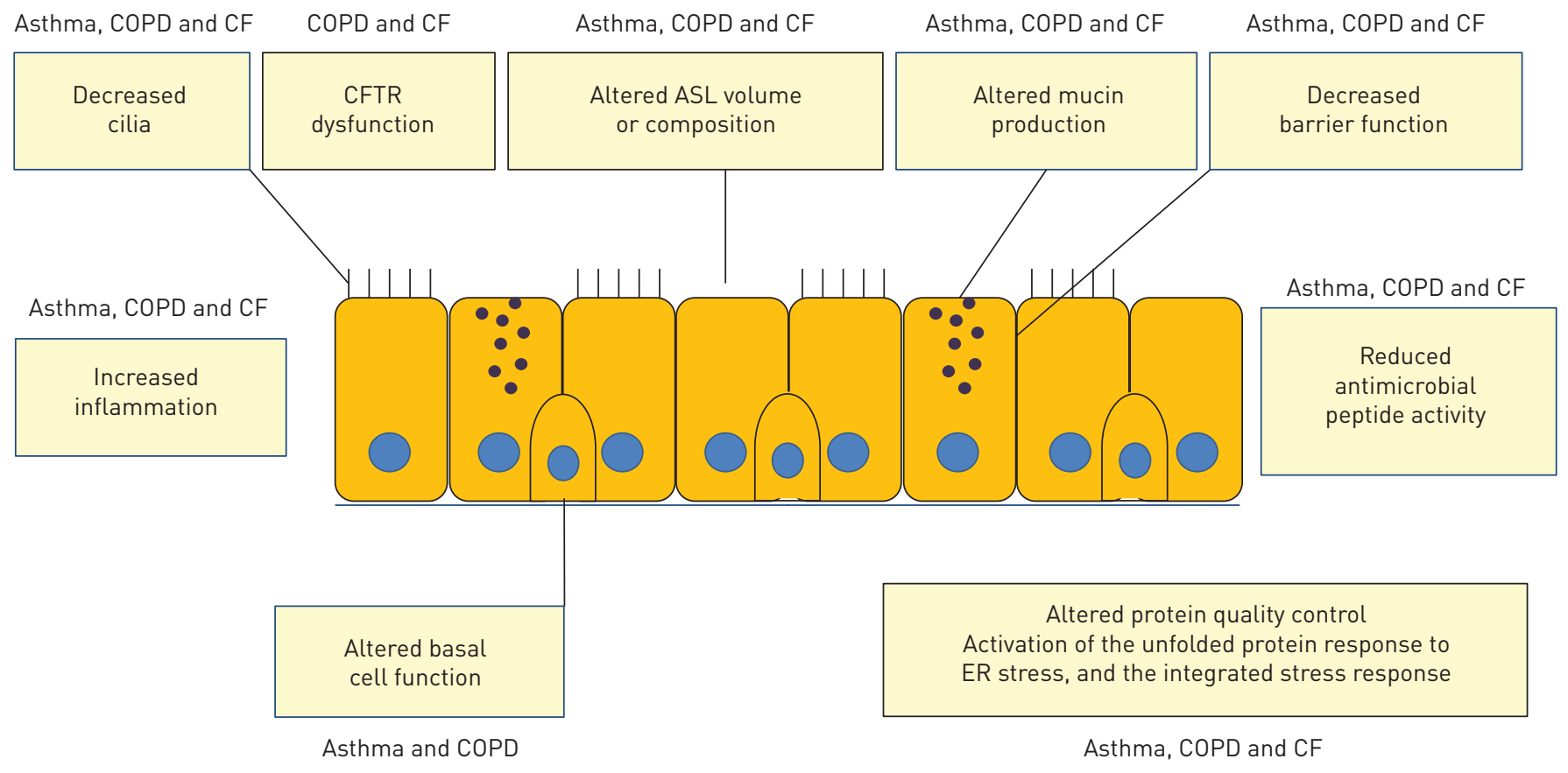

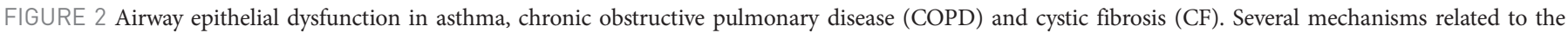

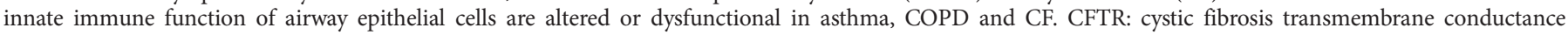
regulator; ASL: airway surface liquid; ER: endoplasmic reticulum. 
function. Additionally, miR-138, through interactions with the transcriptional repressor SIN3A mRNA and other targets, helps regulate both CFTR transcription and post-translational processing [118]. Changes in CFTR abundance and function regulated by microRNAs might help dynamically regulate ASL volume and composition, host defence and mucociliary clearance. The concept of therapeutic modification of microRNAs is now established [119]. As this field advances, there may be opportunities for the development of new therapies aimed at enhancing or reducing the function of specific microRNAs and their targets [120].

\section{Conclusion}

The airway epithelium is more than a physical barrier and is the central player in mucociliary clearance of the lung. The innate immune functions of the epithelium include not only the secretion of a variety of antimicrobial substances, but also cytokines and growth factors that mediate leukocyte recruitment, modulation of adaptive immunity, and tissue repair and remodelling. An increasing number of studies demonstrate that several of these functions are altered or decreased in asthma, COPD and CF (fig. 2). Novel mechanisms that contribute to the dysfunctional airway epithelium are being discovered, and include microRNAs, endoplasmic reticulum stress and the integrated stress response. Furthermore, various features of a dysfunctional innate immune function of AECs appear to persist in culture, indicating that not only acute exposures to inhaled substances or local inflammation affect the epithelium but that genetic and epigenetic mechanisms also contribute. Thus, the development of advanced epithelial cell culture systems and patient-derived epithelial cell cultures are becoming a valuable tool to study pathogenetic mechanisms and novel diagnostics. In addition, these facilitate target identification for drug discovery purposes, as well as evaluation of therapeutic approaches. This is important, because although the anti-inflammatory effects of inhaled steroids may partly restore epithelial cell function in airway diseases, this is by far not optimal. Therefore, other approaches are needed to restore the dysfunctional airway epithelium in these diseases. Such approaches include using vitamin D to boost epithelial defences, CFTR correctors and potentiators, microRNA modification, and small molecules to increase production of antiviral interferons, as well as blocking IL-4 and IL-13.

In summary, our increased understanding of the innate immune functions of the airway epithelium in the healthy lung and in asthma, COPD and CF will contribute to better diagnostics and treatment of patients with these chronic inflammatory diseases. Therefore, current and future basic and translational studies on airway epithelial cell function will probably continue to contribute to meeting the unmet medical needs in the treatment of these diseases with marked morbidity and mortality.

\section{Acknowledgements}

The authors apologise for not being able to include all relevant references due to space limitations.

\section{References}

1 Bals R, Hiemstra PS. Innate immunity in the lung: how epithelial cells fight against respiratory pathogens. Eur Respir J 2004; 23: 327-333.

2 Parker D, Prince A. Innate immunity in the respiratory epithelium. Am J Respir Cell Mol Biol 2011; 45: 189-201.

3 Li Y, Eggermont K, Vanslembrouck V, et al. NKX2-1 activation by SMAD2 signaling after definitive endoderm differentiation in human embryonic stem cell. Stem Cells Dev 2013; 22: 1433-1442.

4 Wong AP, Bear CE, Chin S, et al. Directed differentiation of human pluripotent stem cells into mature airway epithelia expressing functional CFTR protein. Nat Biotechnol 2012; 30: 876-882.

5 Firth AL, Dargitz CT, Qualls SJ, et al. Generation of multiciliated cells in functional airway epithelia from human induced pluripotent stem cells. Proc Natl Acad Sci USA 2014; 111: E1723-E1730.

6 Rock JR, Hogan BL. Epithelial progenitor cells in lung development, maintenance, repair, and disease. Annu Rev Cell Dev Biol 2011; 27: 493-512.

7 Herriges M, Morrisey EE. Lung development: orchestrating the generation and regeneration of a complex organ. Development 2014; 141: 502-513.

8 Wansleeben C, Barkauskas CE, Rock JR, et al. Stem cells of the adult lung: their development and role in homeostasis, regeneration, and disease. Wiley Interdiscip Rev Dev Biol 2013; 2: 131-148.

9 Rock JR, Onaitis MW, Rawlins EL, et al. Basal cells as stem cells of the mouse trachea and human airway epithelium. Proc Natl Acad Sci USA 2009; 106: 12771-12775.

10 Tata PR, Mou H, Pardo-Saganta A, et al. Dedifferentiation of committed epithelial cells into stem cells in vivo. Nature 2013; 503: 218-223.

11 Rupani H, Sanchez-Elsner T, Howarth P. MicroRNAs and respiratory diseases. Eur Respir J 2013; 41: 695-705.

12 Khoshgoo N, Kholdebarin R, Iwasiow BM, et al. MicroRNAs and lung development. Pediatr Pulmonol 2013; 48: 317-323.

13 Harris KS, Zhang Z, McManus MT, et al. Dicer function is essential for lung epithelium morphogenesis. Proc Natl Acad Sci USA 2006; 103: 2208-2213.

14 Williams AE, Larner-Svensson H, Perry MM, et al. MicroRNA expression profiling in mild asthmatic human airways and effect of corticosteroid therapy. PLoS One 2009; 4: e5889.

15 Fulcher ML, Randell SH. Human nasal and tracheo-bronchial respiratory epithelial cell culture. Methods Mol Biol 2013; 945: 109-121. 
Pezzulo AA, Starner TD, Scheetz TE, et al. The air-liquid interface and use of primary cell cultures are important to recapitulate the transcriptional profile of in vivo airway epithelia. Am J Physiol 2011; 300: L25-L31.

17 Dvorak A, Tilley AE, Shaykhiev R, et al. Do airway epithelium air-liquid cultures represent the in vivo airway epithelium transcriptome? Am J Respir Cell Mol Biol 2011; 44: 465-473.

18 Horani A, Dickinson J, Brody S. Applications of mouse airway epithelial cell culture for asthma research. In: Allen IC, ed. Mouse Models of Allergic Disease: Methods and Protocols. Totowa, NJ, Humana Press, 2013; pp. 91-107.

19 Suprynowicz FA, Upadhyay G, Krawczyk E, et al. Conditionally reprogrammed cells represent a stem-like state of adult epithelial cells. Proc Natl Acad Sci USA 2012; 109: 20035-20040.

20 Horani A, Nath A, Wasserman MG, et al. Rho-associated protein kinase inhibition enhances airway epithelial basal-cell proliferation and lentivirus transduction. Am J Respir Cell Mol Biol 2013; 49: 341-347.

21 Gaj T, Gersbach CA, Barbas CF III. ZFN, TALEN, and CRISPR/Cas-based methods for genome engineering. Trends Biotechnol 2013; 31: 397-405.

22 Peterson J, Garges S, Giovanni M, et al. The NIH Human Microbiome Project. Genome Res 2009; 19: 2317-2323.

23 Hansel TT, Johnston SL, Openshaw PJ. Microbes and mucosal immune responses in asthma. Lancet 2013; 381: $861-873$.

24 Dickson RP, Erb-Downward JR, Huffnagle GB. The role of the bacterial microbiome in lung disease. Expert Rev Respir Med 2013; 7: 245-257.

25 Gollwitzer ES, Saglani S, Trompette A, et al. Lung microbiota promotes tolerance to allergens in neonates via PD-L1. Nat Med 2014; 20: 642-647.

26 Sibley CD, Grinwis ME, Field TR, et al. Culture enriched molecular profiling of the cystic fibrosis airway microbiome. PLoS One 2011; 6: e22702.

27 Zemanick ET, Sagel SD, Harris JK. The airway microbiome in cystic fibrosis and implications for treatment. Curr Opin Pediatr 2011; 23: 319-324.

28 Molyneaux PL, Mallia P, Cox MJ, et al. Outgrowth of the bacterial airway microbiome after rhinovirus exacerbation of chronic obstructive pulmonary disease. Am J Respir Crit Care Med 2013; 188: 1224-1231.

29 Song X, Gao H, Lin Y, et al. Alterations in the microbiota drive interleukin-17C production from intestinal epithelial cells to promote tumorigenesis. Immunity 2014; 40: 140-152.

30 Pezzulo AA, Kelly PH, Nassar BS, et al. Abundant DNase I-sensitive bacterial DNA in healthy porcine lungs and its implications for the lung microbiome. Appl Environ Microbiol 2013; 79: 5936-5941.

31 Janeway CA Jr. Approaching the asymptote? Evolution and revolution in immunology. Cold Spring Harb Symp Quant Biol 1989; 54: 1-13.

32 Takeuchi O, Akira S. Pattern recognition receptors and inflammation. Cell 2010; 140: 805-820.

33 O'Neill LA, Golenbock D, Bowie AG. The history of Toll-like receptors - redefining innate immunity. Nat Rev Immunol 2013; 13: 453-460.

34 Anderson KV, Jurgens G, Nusslein-Volhard C. Establishment of dorsal-ventral polarity in the Drosophila embryo: genetic studies on the role of the Toll gene product. Cell 1985; 42: 779-789.

35 Greene CM, McElvaney NG. Toll-like receptor expression and function in airway epithelial cells. Arch Immunol Ther Exp (Warsz) 2005; 53: 418-427.

36 Sha Q, Truong-Tran AQ, Plitt JR, et al. Activation of airway epithelial cells by toll-like receptor agonists. Am J Respir Cell Mol Biol 2004; 31: 358-364.

37 Hess C, Herr C, Beisswenger C, et al. Myeloid RelA regulates pulmonary host defense networks. Eur Respir J 2010; 35: 343-352.

38 Robinson MJ, Sancho D, Slack EC, et al. Myeloid C-type lectins in innate immunity. Nat Immunol 2006; 7: $1258-1265$.

39 Lee HM, Yuk JM, Shin DM, et al. Dectin-1 is inducible and plays an essential role for mycobacteria-induced innate immune responses in airway epithelial cells. J Clin Immunol 2009; 29: 795-805.

40 Sun WK, Lu X, Li X, et al. Dectin-1 is inducible and plays a crucial role in Aspergillus-induced innate immune responses in human bronchial epithelial cells. Eur J Clin Microbiol Infect Dis 2012; 31: 2755-2764.

41 Wang Q, Nagarkar DR, Bowman ER, et al. Role of double-stranded RNA pattern recognition receptors in rhinovirus-induced airway epithelial cell responses. J Immunol 2009; 183: 6989-6997.

42 Lipinski S, Rosenstiel P. Debug your bugs - how NLRs shape intestinal host-microbe interactions. Front Immunol 2013; 4: 479

43 van't Wout EF, Hiemstra PS, Marciniak SJ. The integrated stress response in lung disease. Am J Respir Cell Mol Biol 2014; 50: 1005-1009.

44 Marcinak SJ, Ron D. The unfolded protein response in lung disease. Proc Am Thorac Soc 2010; 7: 356-362.

45 Claudio N, Dalet A, Gatti E, et al. Mapping the crossroads of immune activation and cellular stress response pathways. EMBO J 2013; 32: 1214-1224.

46 Blander JM, Sander LE. Beyond pattern recognition: five immune checkpoints for scaling the microbial threat. Nat Rev Immunol 2012; 12: 215-225.

47 Lee RJ, Kofonow JM, Rosen PL, et al. Bitter and sweet taste receptors regulate human upper respiratory innate immunity. J Clin Invest 2014; 124: 1393-1405.

48 Fahy JV, Dickey BF. Airway mucus function and dysfunction. N Engl J Med 2010; 363: $2233-2247$.

49 Thornton DJ, Rousseau K, McGuckin MA. Structure and function of the polymeric mucins in airways mucus. Annu Rev Physiol 2008; 70: 459-486.

50 Roy MG, Livraghi-Butrico A, Fletcher AA, et al. Muc5b is required for airway defence. Nature 2014; 505: 412-416.

51 Adler KB, Tuvim MJ, Dickey BF. Regulated mucin secretion from airway epithelial cells. Front Endocrinol (Lausanne) 2013; 4; 129.

52 Button B, Cai LH, Ehre C, et al. A periciliary brush promotes the lung health by separating the mucus layer from airway epithelia. Science 2012; 337: 937-941.

53 Mall MA, Button B, Johannesson B, et al. Airway surface liquid volume regulation determines different airway phenotypes in liddle compared with $\beta E N a C$-overexpressing mice. J Biol Chem 2010; 285: 26945-26955.

54 Pezzulo AA, Tang XX, Hoegger MJ, et al. Reduced airway surface $\mathrm{pH}$ impairs bacterial killing in the porcine cystic fibrosis lung. Nature 2012; 487: 109-113. 
Hoegger MJ, Fischer AJ, McMenimen JD, et al. Cystic fibrosis. Impaired mucus detachment disrupts mucociliary transport in a piglet model of cystic fibrosis. Science 2014; 345: 818-822.

Wain LV, Odenthal-Hesse L, Abujaber R, et al. Copy number variation of the $\beta$-defensin genes in Europeans: no supporting evidence for association with lung function, chronic obstructive pulmonary disease or asthma. PLoS One 2014; 9: e84192.

Moser C, Weiner DJ, Lysenko E, et al. $\beta$-Defensin 1 contributes to pulmonary innate immunity in mice. Infect Immun 2002; 70: 3068-3072.

Morrison G, Kilanowski F, Davidson D, et al. Characterization of the mouse $\beta$ defensin 1, Defb1, mutant mouse model. Infect Immun 2002; 70: 3053-3060.

Herr C, Beisswenger C, Hess C et al. Suppression of pulmonary innate host defence in smokers. Thorax 2009; 64: 144-149.

Bals R, Wang X, Zasloff M, et al. The peptide antibiotic LL-37/hCAP-18 is expressed in epithelia of the human lung where it has broad antimicrobial activity at the airway surface. Proc Natl Acad Sci USA 1998; 95: 9541-9546. Kovach MA, Ballinger MN, Newstead MW, et al. Cathelicidin-related antimicrobial peptide is required for effective lung mucosal immunity in Gram-negative bacterial pneumonia. J Immunol 2012; 189: 304-311.

Kahlenberg JM, Kaplan MJ. Little peptide, big effects: the role of LL-37 in inflammation and autoimmune disease. J Immunol 2013; 191: 4895-4901.

Liu PT, Stenger S, Li H, et al. Toll-like receptor triggering of a vitamin D-mediated human antimicrobial response. Science 2006; 311: 1770-1773.

Felgentreff $\mathrm{K}$, Beisswenger $\mathrm{C}$, Griese $\mathrm{M}$, et al. The antimicrobial peptide cathelicidin interacts with airway mucus. Peptides 2006; 27: 3100-3106.

Brinkmann V, Reichard U, Goosmann C, et al. Neutrophil extracellular traps kill bacteria. Science 2004; 303: 1532-1535.

Conner GE, Salathe M, Forteza R. Lactoperoxidase and hydrogen peroxide metabolism in the airway. Am J Respir Crit Care Med 2002; 166: S57-S61.

Moskwa P, Lorentzen D, Excoffon KJ, et al. A novel host defense system of airways is defective in cystic fibrosis. Am J Respir Crit Care Med 2007; 175: 174-183.

Wink DA, Hines HB, Cheng RY, et al. Nitric oxide and redox mechanisms in the immune response. J Leukoc Biol 2011; 89: 873-891.

Vareille M, Kieninger E, Edwards MR, et al. The airway epithelium: soldier in the fight against respiratory viruses. Clin Microbiol Rev 2011; 24: 210-229.

Holtzman M, Patel D, Kim HJ, et al. Hypersusceptibility to respiratory viruses as a shared mechanism for asthma, chronic obstructive pulmonary disease, and cystic fibrosis. Am J Respir Cell Mol Biol 2011; 44: 739-742.

Patel DA, Patel AC, Nolan WC, et al. High throughput screening for small molecule enhancers of the interferon signaling pathway to drive next-generation antiviral drug discovery. PLoS One 2012; 7: e36594.

Zheng S, De BP, Choudhary S, et al. Impaired innate host defense causes susceptibility to respiratory virus infections in cystic fibrosis. Immunity 2003; 18: 619-630.

Yang Z, Klionsky DJ. Eaten alive: a history of macroautophagy. Nat Cell Biol 2010; 12: 814-822. Autophagy 2014; 10: 532-534.

Heijink IH, Nawijn MC, Hackett TL. Airway epithelial barrier function regulates the pathogenesis of allergic asthma. Clin Exp Allergy 2014; 44: 620-630.

Grainge CL, Davies DE. Epithelial injury and repair in airways diseases. Chest 2013; 144: 1906-1912.

Caputo A, Caci E, Ferrera L, et al. TMEM16A, a membrane protein associated with calcium-dependent chloride channel activity. Science 2008; 322: 590-594.

Anagnostopoulou P, Riederer B, Duerr J, et al. SLC26A9-mediated chloride secretion prevents mucus obstruction in airway inflammation. J Clin Invest 2012; 122: 3629-3634.

Huang YJ, Boushey HA. The microbiome and asthma. Ann Am Thorac Soc 2014; 11: S48-S51.

Wark PA, Johnston SL, Bucchieri F, et al. Asthmatic bronchial epithelial cells have a deficient innate immune response to infection with rhinovirus. J Exp Med 2005; 201: 937-947.

Beisswenger C, Kandler K, Hess C, et al. Allergic airway inflammation inhibits pulmonary antibacterial host defense. J Immunol 2006; 177: 1833-1837.

Byers DE, Alexander-Brett J, Patel AC, et al. Long-term IL-33-producing epithelial progenitor cells in chronic obstructive lung disease. J Clin Invest 2013; 123: 3967-3982.

Grainge CL, Lau LC, Ward JA, et al. Effect of bronchoconstriction on airway remodeling in asthma. $N$ Engl Med 2011; 364: 2006-2015.

aumann F, Elssner A, Mazur G, et al. Transforming growth factor- $\beta 1$ is a potent inhibitor of secretory leukoprotease inhibitor expression in a bronchial epithelial cell line. Munich Lung Transplant Group. Eur Respir J 2000; 15: 1052-1057.

Bedke N, Sammut D, Green B, et al. Transforming growth factor- $\beta$ promotes rhinovirus replication in bronchial epithelial cells by suppressing the innate immune response. PLoS One 2012; 7: e44580.

Schamberger AC, Mise N, Jia J, et al. Cigarette smoke-induced disruption of bronchial epithelial tight junctions is prevented by transforming growth factor- $\beta$. Am J Respir Cell Mol Biol 2014; 50: 1040-1052.

Jono $\mathrm{H}$, Xu $\mathrm{H}$, Kai $\mathrm{H}$, et al. Transforming growth factor- $\beta$-Smad signaling pathway negatively regulates nontypeable Haemophilus influenzae-induced MUC5AC mucin transcription via mitogen-activated protein kinase (MAPK) phosphatase-1-dependent inhibition of p38 MAPK. J Biol Chem 2003; 278: 27811-27819.

Harrop CA, Gore RB, Evans CM, et al. TGF- $\beta_{2}$ decreases baseline and IL-13-stimulated mucin production by primary human bronchial epithelial cells. Exp Lung Res 2013; 39: 39-47.

Proud D, Hudy MH, Wiehler S, et al. Cigarette smoke modulates expression of human rhinovirus-induced airway epithelial host defense genes. PLoS One 2012; 7: e40762.

Rusznak C, Mills PR, Devalia JL, et al. Effect of cigarette smoke on the permeability and IL-1 $\beta$ and sICAM-1 release from cultured human bronchial epithelial cells of never-smokers, smokers, and patients with chronic obstructive pulmonary disease. Am J Respir Cell Mol Biol 2000; 23: 530-536. 
Kilsgard O, Andersson P, Malmsten M, et al. Peptidylarginine deiminases present in the airways during tobacco smoking and inflammation can citrullinate the host defense peptide LL-37, resulting in altered activities. Am J Respir Cell Mol Biol 2012; 46: 240-248.

Nyunoya T, Mebratu Y, Contreras A, et al. Molecular processes that drive cigarette smoke-induced epithelial cell fate of the lung. Am J Respir Cell Mol Biol 2013; 50: 471-482.

Steiling K, van den Berge M, Hijazi K, et al. A dynamic bronchial airway gene expression signature of chronic obstructive pulmonary disease and lung function impairment. Am J Respir Crit Care Med 2013; 187: 933-942.

Mall MA, Hartl D. CFTR: cystic fibrosis and beyond. Eur Respir J 2014; 44: 1042-1054.

Schulz C, Wolf K, Harth M, et al. Expression and release of interleukin- 8 by human bronchial epithelial cells from patients with chronic obstructive pulmonary disease, smokers, and never-smokers. Respiration 2003; 70 : 254-261.

van't Wout EF, Dickens JA, van Schadewijk A, et al. Increased ERK signalling promotes inflammatory signalling in primary airway epithelial cells expressing Z alphal-antitrypsin. Hum Mol Genet 2014; 23: 929-941.

Rogers CS, Stoltz DA, Meyerholz DK, et al. Disruption of the CFTR gene produces a model of cystic fibrosis in newborn pigs. Science 2008; 321: 1837-1841.

Ostedgaard LS, Meyerholz DK, Chen JH, et al. The $\triangle$ F508 mutation causes CFTR misprocessing and cystic fibrosis-like disease in pigs. Sci Transl Med 2011; 3: 74ra24.

Sun X, Yan Z, Yi Y, et al. Adeno-associated virus-targeted disruption of the CFTR gene in cloned ferrets. $J$ Clin Invest 2008; 118: 1578-1583.

Tuggle KL, Birket SE, Cui X, et al. Characterization of defects in ion transport and tissue development in cystic fibrosis transmembrane conductance regulator (CFTR)-knockout rats. PLoS One 2014; 9: e91253.

Stoltz DA, Meyerholz DK, Pezzulo AA, et al. Cystic fibrosis pigs develop lung disease and exhibit defective bacterial eradication at birth. Sci Transl Med 2010; 2: 29-31.

Sun X, Sui H, Fisher JT, et al. Disease phenotype of a ferret CFTR-knockout model of cystic fibrosis. J Clin Invest 2010; 120: 3149-3160. conductance regulator-knockout ferrets. Am J Respir Cell Mol Biol 2014; 50: 502-512.

McShane D, Davies JC, Davies MG, et al. Airway surface pH in subjects with cystic fibrosis. Eur Respir J 2003; 21: 37-42.

Abou Alaiwa MH, Beer AM, Pezzulo AA, et al. Neonates with cystic fibrosis have a reduced nasal liquid pH: a small pilot study. J Cyst Fibros 2014; 13: 373-377.

Berkebile AR, McCray PB Jr. Effects of airway surface liquid pH on host defense in cystic fibrosis. Int J Biochem Cell Biol 2014; 52: 124-129.

Boucher RC. Airway surface dehydration in cystic fibrosis: pathogenesis and therapy. Annu Rev Med 2007; 58: 157-170.

Hobbs CA, Da Tan C, Tarran R. Does epithelial sodium channel hyperactivity contribute to cystic fibrosis lung disease? J Physiol 2013; 591: 4377-4387.

Chen JH, Stoltz DA, Karp PH, et al. Loss of anion transport without increased sodium absorption characterizes newborn porcine cystic fibrosis airway epithelia. Cell 2010; 143: 911-923.

Fisher JT, Tyler SR, Zhang Y, et al. Bioelectric characterization of epithelia from neonatal CFTR knockout ferrets. Am J Respir Cell Mol Biol 2013; 49: 837-844.

Itani OA, Chen JH, Karp $\mathrm{PH}$, et al. Human cystic fibrosis airway epithelia have reduced $\mathrm{Cl}^{-}$conductance but not increased $\mathrm{Na}^{+}$conductance. Proc Natl Acad Sci USA 2011; 108: 10260-10265.

Mall M, Grubb BR, Harkema JR, et al. Increased airway epithelial $\mathrm{Na}^{+}$absorption produces cystic fibrosis-like lung disease in mice. Nat Med 2004; 10: 487-493.

Henderson AG, Ehre C, Button B, et al. Cystic fibrosis airway secretions exhibit mucin hyperconcentration and increased osmotic pressure. J Clin Invest 2014; 124: 3047-3060.

Oglesby IK, Bray IM, Chotirmall SH, et al. miR-126 is downregulated in cystic fibrosis airway epithelial cells and regulates TOM1 expression. J Immunol 2010; 184: 1702-1709.

Gillen AE, Gosalia N, Leir SH, et al. MicroRNA regulation of expression of the cystic fibrosis transmembrane conductance regulator gene. Biochem J 2011; 438: 25-32.

Megiorni F, Cialfi S, Dominici C, et al. Synergistic post-transcriptional regulation of the cystic fibrosis transmembrane conductance regulator (CFTR) by miR-101 and miR-494 specific binding. PLoS One 2011; 6: e26601.

7 Ramachandran S, Karp PH, Osterhaus SR, et al. Post-transcriptional regulation of cystic fibrosis transmembrane conductance regulator expression and function by microRNAs. Am J Respir Cell Mol Biol 2013; 49: 544-551.

Ramachandran S, Karp PH, Jiang P, et al. A microRNA network regulates expression and biosynthesis of wild-type and DeltaF508 mutant cystic fibrosis transmembrane conductance regulator. Proc Natl Acad Sci USA 2012; 109: 13362-13367.

9 Greene CM, Gaughan KP. microRNAs in asthma: potential therapeutic targets. Curr Opin Pulm Med 2013; 19: $66-72$.

Jackson AL, Levin AA. Developing microRNA therapeutics: approaching the unique complexities. Nucleic Acid Ther 2012; 22: 213-225. 